\title{
CHARACTERIZATION OF ADJOINTABLE OPERATORS ON HILBERT $C^{*}$-modules
}

\author{
Sayed Khalil Ekrami ${ }^{1}$, Madjid Mirzavaziri ${ }^{2}$ \\ ${ }^{1}$ Department of Mathematics \\ Payame Noor University \\ P.O. Box 19395-3697, Tehran, IRAN \\ ${ }^{2}$ Department of Pure Mathematics \\ Ferdowsi University of Mashhad \\ P. O. Box 1159, Mashhad 91775, IRAN
}

\begin{abstract}
Let $\mathcal{H}$ be a Hilbert $C^{*}$-module over a unital $C^{*}$-algebra $\mathcal{A}$. In this paper, we find the general form of the mappings $T: \mathcal{H} \rightarrow \mathcal{H}$ satisfing

$$
2\langle T(x), T(y)\rangle=\langle T(x), y\rangle+\langle x, T(y)\rangle \quad(x, y \in \mathcal{H}),
$$

as adjointable (bounded) $\mathcal{A}$-linear operators. The generalized Hyers-Ulam stability of the functional equation is discussed.
\end{abstract}

AMS Subject Classification: 39B52, 46L08

Key Words: Hilbert $C^{*}$-module, $C^{*}$-algebra, $\mathcal{A}$-linear mapping

\section{Introduction and Preliminaries}

The notion of a Hilbert $C^{*}$-module initiated by Kaplansky [3] as a generalization of a Hilbert space in which the inner product takes its values in a $C^{*}$-algebra.

Let $\mathcal{A}$ be a $C^{*}$-algebra. A pre-Hilbert $\mathcal{A}$-module or an inner product $\mathcal{A}$ module is a complex linear space $\mathcal{H}$ which is a left $\mathcal{A}$-module with compatible scalar multiplication $\lambda(a x)=(\lambda a) x=a(\lambda x) \quad(\lambda \in \mathbb{C}, x \in \mathcal{H}, a \in \mathcal{A})$, together with an $\mathcal{A}$-valued inner product $(x, y) \mapsto\langle x, y\rangle: \mathcal{H} \times \mathcal{H} \rightarrow \mathcal{A}$ such that for each $x, y, z \in \mathcal{H}, \alpha, \beta \in \mathbb{C}$ and $a \in \mathcal{A}$,

\begin{tabular}{llc}
\hline Received: & September 6, 2015 & (C) 2016 Academic Publications, Ltd. \\
Published: & February 3, 2016 & url: www.acadpubl.eu
\end{tabular}

${ }^{\S}$ Correspondence author 
(i) $\langle x, x\rangle \geq 0$ and the equality holds if and only if $x=0$.

(ii) $\langle\alpha x+\beta y, z\rangle=\alpha\langle x, z\rangle+\beta\langle y, z\rangle$,

(iii) $\langle a x, y\rangle=a\langle x, y\rangle$,

(iv) $\langle x, y\rangle^{*}=\langle y, x\rangle$.

The notion of a right Hilbert $\mathcal{A}$-module can be defined similarly. Note that the condition (i) is understood as a statement in the $C^{*}$-algebra $\mathcal{A}$, where an element $a$ is called positive if it can be represented as $b b^{*}$ for some $b \in \mathcal{A}$. The conditions (ii) and (iv) impliy the inner product to be conjugate-linear in its second variable. Validity of a useful version of the classical Cauchy-Schwartz inequality follows that $\|x\|=\|\langle x, x\rangle\|^{\frac{1}{2}}$ defines a norm on $\mathcal{H}$ making it into a normed left $\mathcal{A}$-module. An inner product $\mathcal{A}$-module $\mathcal{H}$ which is complete with respect to the norm $\|x\|$ is called a Hilbert $\mathcal{A}$-module or a Hilbert $C^{*}$-module over the $C^{*}$-algebra $\mathcal{A}$. Every $C^{*}$-algebra $\mathcal{A}$ is a Hilbert $\mathcal{A}$-module under the $\mathcal{A}$-valued inner product $\langle a, b\rangle=a^{*} b \quad(a, b \in A)$. Every complex Hilbert space is a left Hilbert $\mathbb{C}$-module.

One may define an $\mathcal{A}$-valued norm $|x|=\langle x, x\rangle^{\frac{1}{2}}$ (where, $|a|$ denotes the unique square root of the positive element $a$ in $\mathcal{A}$ ). Clearly, $\|x\|\|=\| x \|$, for each $x \in \mathcal{H}$. The $\mathcal{A}$-valued norm $|x|$ is a useful device but it needs to be handled with care. For example, it is known that $|$.$| does not satisfy the triangle$ inequality $|x+y| \leq|x|+|y|$ for each $x, y \in \mathcal{H}$; cf. [4].

Let $\mathcal{H}$ be a Hilbert $C^{*}$-module over a $C^{*}$-algebra $\mathcal{A}$. A system $\left(e_{i}\right)_{i \in I}$ in $\mathcal{H}$ is called orthogonal, if $\left\langle e_{i}, e_{j}\right\rangle=0$ whenever $i \neq j$. An orthogonal system $\left(e_{i}\right)_{i \in I}$ in $\mathcal{H}$ is said to be an orthonormal, provided $\mathcal{A}$ is unital and for the inner squares it happens that $\left\langle e_{i}, e_{i}\right\rangle=1$ for all $i \in I$. Let $\left(e_{i}\right)_{i \in I}$ be an orthonormal system in a Hilbert module $\mathcal{H}$ over a unital $C^{*}$-algebra $\mathcal{A}$. Landi and Pavlov showed in Theorem 2.10 of [5] that the following conditions are equivalent:

(i) For any $x$ of $\mathcal{H}$ there are elements $a_{i}$ of $\mathcal{A}$ such that

$$
x=\sum_{i \in I} a_{i} e_{i}
$$

where convergence in norm is meant and

$$
\sum_{i \in I} a_{i} e_{i}=\lim _{F \in \mathcal{F}} \sum_{i \in F} a_{i} e_{i}
$$

indicates the limit over the set $\mathcal{F}$ of all finite subsets of $I$, directed by inclusions. 
(ii) The system $\left(e_{i}\right)_{i \in I}$ generates $\mathcal{H}$ over $\mathcal{A}$, that is to say, the closure of its $\mathcal{A}$-linear span coincides with $\mathcal{H}$.

(iii) The system $\left(e_{i}\right)_{i \in I}$ is closed, that is to say, for any $x \in \mathcal{H}$

$$
\langle x, x\rangle=\sum_{i \in I}\left\langle x, e_{i}\right\rangle\left\langle e_{i}, x\right\rangle
$$

where the series converges in norm.

An orthonormal system $\left(e_{i}\right)_{i \in I}$ satisfying the equivalent conditions (i)-(iii) is called a Schauder basis for $\mathcal{H}$ over $\mathcal{A}$. If $\left(e_{i}\right)_{i \in I}$ is a Schauder basis for $\mathcal{H}$, then the coefficients in the decomposition (1) are unique for any vector $x$ of $\mathcal{H}$. In fact for any $i \in I, a_{i}=\left\langle x, e_{i}\right\rangle$. Thus any vector of $\mathcal{H}$ is the limit in norm of its Fourier series. Any Schauder basis $\left(e_{i}\right)_{i \in I}$ is complete, i.e. there is no non-zero vector $x$ of $\mathcal{H}$ such that $\left\langle x, e_{i}\right\rangle=0$ for all $i \in I$. Note that by Proposition 3.1. of [5] any two closed orthonormal systems of a Hilbert module over a unital $C^{*}$-algebra have the same cardinality.

Let $\mathcal{H}$ and $\mathcal{K}$ be Hilbert $C^{*}$-modules over a $C^{*}$-algebra $\mathcal{A}$. A mapping $T: \mathcal{H} \rightarrow \mathcal{K}$ is said to be $\mathcal{A}$-linear, if

$$
T(a x+\lambda y)=a T(x)+\lambda T(y)
$$

for all $x, y \in \mathcal{H}, a \in \mathcal{A}$ and $\lambda \in \mathbb{C}$. A mapping $T: \mathcal{H} \rightarrow \mathcal{K}$ is said to be adjointable, if there exists a mapping $S: \mathcal{K} \rightarrow \mathcal{H}$ such that

$$
\langle T(x), y\rangle=\langle x, S(y)\rangle
$$

for all $x \in D_{T} \subseteq \mathcal{H}, y \in D_{S} \subseteq \mathcal{K}$. The unique mapping $S$ is denoted by $T^{*}$ and is called the adjoint of $T$. It is well known that any adjointable mapping $T: \mathcal{H} \rightarrow \mathcal{K}$ is $\mathcal{A}$-linear and bounded. $L(\mathcal{H}, \mathcal{K})$, the set of adjointable maps from $\mathcal{H}$ to $\mathcal{K}$ is a $C^{*}$-algebra [4]. The $C^{*}$-algebra of adjointable maps from $\mathcal{H}$ to $\mathcal{H}$ is denoted by $L(\mathcal{H})$.

In general, bounded $\mathcal{A}$-linear operator may fail to possess an adjoint (cf., [4]). However, if $\mathcal{H}$ is a Hilbert $C^{*}$-module over the $C^{*}$-algebra $\mathcal{K}=K(H)$ of all compact operators on a Hilbert space $H$, then (with another concept of orthonormal basis for Hilbert $C^{*}$-modules) D. Bakić and B. Guljaš ([1], Remark $5)$ showed that each bounded $\mathcal{K}$-linear operator on $\mathcal{H}$ is necessarily adjointable.

In 2003, Radu [6] employed the following result, due to Diaz and Margolis [2], to prove the stability of a Cauchy functional equation. 
Proposition 1.1. (The fixed point alternative principle). Let $(X, d)$ be a generalized complete metric space and $J: X \rightarrow X$ be a strictly contractive mapping; that is

$$
d(J(x), J(y)) \leq L d(x, y) \quad(x, y \in X)
$$

for some (Lipschitz) constant $0<L<1$. Then, for a given element $x \in X$, exactly one of the following assertions is true: either

(a) $d\left(J^{n} x, J^{n+1} x\right)=\infty$ for all $n \geq 0$, or

(b) there exists some integer $n_{0}$ such that $d\left(J^{n} x, J^{n+1} x\right)<\infty$ for all $n \geq n_{0}$.

Actually, if (b) holds, then

$\left(b_{1}\right)$ the sequence $\left\{J^{n} x\right\}$ converges to a fixed point $x^{*}$ of $J$,

$\left(b_{2}\right) x^{*}$ is the unique fixed point of $J$ in $X_{0}:=\left\{y \in X ; d\left(J^{n_{0}} x, y\right)<\infty\right\}$;

$\left(b_{3}\right) d\left(y, x^{*}\right) \leq \frac{1}{1-L} d(y, J y)$ for all $y \in X_{0}$.

Let $\mathcal{H}$ be a Hilbert $C^{*}$-module over a $C^{*}$-algebra $\mathcal{A}$ and $T: \mathcal{H} \rightarrow \mathcal{H}$ be a mapping. In this paper, we introduce the new functional equation

$$
2\langle T(x), T(y)\rangle=\langle T(x), y\rangle+\langle x, T(y)\rangle \quad(x, y \in \mathcal{H})
$$

In the first section, we show that $T$ is a bounded $\mathcal{A}$-linear mapping and we find all of such bounded $\mathcal{A}$-linear mappings. Also we show that $T$ is adjointable. We will show that the unique inner product preserving solution of the functional equation $(\diamond)$, is the identity function.

In the next section, we consider the generalized Hyers-Ulam stability for the above functional equation. We use the fixed point alternative theorem to show that if $\mathcal{H}$ is a Hilbert $C^{*}$-modules over a $C^{*}$-algebra $\mathcal{A}$ and $f: \mathcal{H} \rightarrow \mathcal{H}$ satisfies the inequality

$$
\|2\langle f(x), f(y)\rangle-\langle f(x), y\rangle-\langle x, f(y)\rangle\| \leq \varphi(x, y) \quad(x, y \in \mathcal{H})
$$

then under suitable conditions on $\varphi: \mathcal{H} \times \mathcal{H} \rightarrow[0, \infty)$, there is a unique adjointable $\mathcal{A}$-linear mapping $T: \mathcal{H} \rightarrow \mathcal{H}$, which suitably approximates $f$. 


\section{General Solution}

In this section, we show that a function $T: \mathcal{H} \rightarrow \mathcal{H}$ satisfies $(\diamond)$, if and only if, $T$ is an adjointable (bounded) $\mathcal{A}$-linear operator satisfying the equation $2 T^{*} T=T+T^{*}$.

Theorem 2.1. Let $\mathcal{H}$ be a Hilbert $C^{*}$-module over a $C^{*}$-algebra $\mathcal{A}$. Any mapping $T: \mathcal{H} \rightarrow \mathcal{H}$ satisfying $(\diamond)$ is a bounded $\mathcal{A}$-linear operator with $\|T\| \leq$ 1.

Proof. It follows from $(\diamond)$ that

$$
\begin{aligned}
& 2\langle T(x+y)-T(x)-T(y), T(z)\rangle \\
& =2\langle T(x+y), T(z)\rangle-2\langle T(x), T(z)\rangle-2\langle T(y), T(z)\rangle \\
& =\langle T(x+y), z\rangle+\langle x+y, T(z)\rangle \\
& -\langle T(x), z\rangle-\langle x, T(z)\rangle-\langle T(y), z\rangle-\langle y, T(z)\rangle \\
& =\langle T(x+y)-T(x)-T(y), z\rangle
\end{aligned}
$$

for all $x, y, z \in \mathcal{H}$, which implies that

$$
\langle T(x+y)-T(x)-T(y), T(z)\rangle=\left\langle T(x+y)-T(x)-T(y), \frac{z}{2}\right\rangle
$$

for all $x, y, z \in \mathcal{H}$. It follows from (2) that

$$
\begin{aligned}
& \langle T(x+y)-T(x)-T(y), T(x+y)-T(x)-T(y)\rangle \\
& =\langle T(x+y)-T(x)-T(y), T(x+y)\rangle \\
& -\langle T(x+y)-T(x)-T(y), T(x)\rangle \\
& -\langle T(x+y)-T(x)-T(y), T(y)\rangle \\
& =\left\langle T(x+y)-T(x)-T(y), \frac{x+y}{2}-\frac{x}{2}-\frac{y}{2}\right\rangle=0
\end{aligned}
$$

for all $x, y \in \mathcal{H}$. From the above equation, we get

$$
T(x+y)=T(x)+T(y)
$$

for all $x, y \in \mathcal{H}$. Hence $T$ is an additive mapping. 
From

$$
\begin{aligned}
& 2\langle T(a x)-a T(x), T(a x)-a T(x)\rangle \\
& =2\langle T(a x), T(a x)\rangle-2\langle a T(x), T(a x)\rangle \\
& -2\langle T(a x), a T(x)\rangle+2\langle a T(x), a T(x)\rangle \\
& =\langle T(a x), x\rangle a^{*}+a\langle x, T(a x)\rangle-a\langle T(x), x\rangle a^{*}-a\langle x, T(a x)\rangle \\
& -\langle T(a x), x\rangle a^{*}-a\langle x, T(x)\rangle a^{*}+a\langle T(x), x\rangle a^{*}+a\langle x, T(x)\rangle a^{*} \\
& =0
\end{aligned}
$$

for all $a \in \mathcal{A}$ and $x \in \mathcal{H}$, we deduce that $T(a x)=a T(x)$. In the same manner, we deduce that $T(\lambda x)=\lambda T(x)$ for all $x \in \mathcal{H}$ and $\lambda \in \mathbb{C}$. Thus

$$
T(a x+\lambda y)=a T(x)+\lambda T(y)
$$

for all $x, y \in \mathcal{H}, a \in \mathcal{A}$ and $\lambda \in \mathbb{C}$, i.e. $T$ is an $\mathcal{A}$-linear operator.

Putting $x=y$ in $(\diamond)$, we get

$$
2\langle T(x), T(x)\rangle=\langle T(x), x\rangle+\langle x, T(x)\rangle \quad(x \in \mathcal{H}) .
$$

Thus

$$
\begin{aligned}
2\|T(x)\|^{2} & =\left.2\|\| T(x)\right|^{2}\|=2\|\langle T(x), T(x)\rangle\|=\|\langle T(x), x\rangle+\langle x, T(x)\rangle \| \\
& \leq 2\|T(x)\|\|x\| \quad(x \in \mathcal{H}) .
\end{aligned}
$$

and so

$$
\|T\|^{2}=\sup _{\|x\| \leq 1}\|T(x)\|^{2} \leq \sup _{\|x\| \leq 1}\|T(x)\|\|x\|=\|T\|
$$

which implies that $\|T\| \leq 1$. This completes the proof.

Lemma 2.2. An $\mathcal{A}$-linear operator $T: \mathcal{H} \rightarrow \mathcal{H}$ satisfies $(\diamond)$, if and only if $T$ satisfies

$$
2\left\langle T\left(e_{i}\right), T\left(e_{j}\right)\right\rangle=\left\langle T\left(e_{i}\right), e_{j}\right\rangle+\left\langle e_{i}, T\left(e_{j}\right)\right\rangle \quad(i, j \in I) .
$$

Proof. If $T$ satisfies $(\diamond)$, then trivially $T$ satisfies (4). Assume that $T$ satisfies (4), and let $x, y \in \mathcal{H}$. Then $x=\sum_{i \in I} a_{i} e_{i}$ and $y=\sum_{j \in I} b_{j} e_{j}$, where $a_{i}=\left\langle x, e_{i}\right\rangle$ and $b_{j}=\left\langle y, e_{j}\right\rangle$ for $i, j \in I$. It follows from Theorem 2.1 that

$$
2\langle T(x), T(y)\rangle=2\left\langle T\left(\sum_{i \in I} a_{i} e_{i}\right), T\left(\sum_{j \in I} b_{j} e_{j}\right)\right\rangle
$$




$$
\begin{aligned}
& =\sum_{i \in I} \sum_{j \in I} a_{i}\left(2\left\langle T\left(e_{i}\right), T\left(e_{j}\right)\right\rangle\right) b_{j}^{*} \\
& =\sum_{i \in I} \sum_{j \in I} a_{i}\left(\left\langle T\left(e_{i}\right), e_{j}\right\rangle+\left\langle e_{i}, T\left(e_{j}\right)\right\rangle\right) b_{j}^{*} \\
& =\sum_{i \in I} \sum_{j \in I} a_{i}\left\langle T\left(e_{i}\right), e_{j}\right\rangle b_{j}^{*}+\sum_{i \in I} \sum_{j \in I} a_{i}\left\langle e_{i}, T\left(e_{j}\right)\right\rangle b_{j}^{*} \\
& =\left\langle T\left(\sum_{i \in I} a_{i} e_{i}\right), \sum_{j \in I} b_{j} e_{j}\right\rangle+\left\langle\sum_{i \in I} a_{i} e_{i}, T\left(\sum_{j \in I} b_{j} e_{j}\right)\right\rangle \\
& =\langle T(x), y\rangle+\langle x, T(y)\rangle .
\end{aligned}
$$

Let $\left(e_{i}\right)_{i \in I}$ be a Schauder basis for Hilbert $C^{*}$-module $\mathcal{H}$ over a unital $C^{*}$ algebra $\mathcal{A}$ and $T: \mathcal{H} \rightarrow \mathcal{H}$ be an $\mathcal{A}$-linear operator. Let $a_{i j}=\left\langle T\left(e_{j}\right), e_{i}\right\rangle$ for all $i, j \in I$ and consider the matrix $A=\left[a_{i j}\right]$ corresponding to the $\mathcal{A}$-linear operator $T$. The next theorem characterizes the bounded $\mathcal{A}$-linear operators $T$ satisfying $(\diamond)$.

Theorem 2.3. Let $\mathcal{H}$ be a Hilbert $C^{*}$-module over a unital $C^{*}$-algebra $\mathcal{A}$. An $\mathcal{A}$-linear operator $T: \mathcal{H} \rightarrow \mathcal{H}$ satisfies $(\diamond)$, if and only if,

$$
2 A^{t} A^{*}=A^{t}+A^{*} \text {. }
$$

where $A^{t}$ is the transpose of $A$ and $A^{*}=\left[a_{i j}^{*}\right]$.

Proof. Suppose that $T$ satisfies $(\diamond)$, then from Lemma 2.2, $T$ satisfies (4) for all $i, j \in I$. Since for all $i \in I, T\left(e_{i}\right)=\sum_{p \in I}\left\langle T\left(e_{i}\right), e_{p}\right\rangle e_{p}$, then we have

$$
\begin{aligned}
& 2\left\langle\sum_{p \in I}\left\langle T\left(e_{i}\right), e_{p}\right\rangle e_{p}, \sum_{q \in I}\left\langle T\left(e_{j}\right), e_{q}\right\rangle e_{q}\right\rangle \\
& =\left\langle\sum_{p \in I}\left\langle T\left(e_{i}\right), e_{p}\right\rangle e_{p}, e_{j}\right\rangle+\left\langle e_{i}, \sum_{q \in I}\left\langle T\left(e_{j}\right), e_{q}\right\rangle e_{q}\right\rangle .
\end{aligned}
$$

which implies that

$$
\begin{aligned}
& 2 \sum_{p \in I} \sum_{q \in I}\left\langle T\left(e_{i}\right), e_{p}\right\rangle\left\langle e_{p}, e_{q}\right\rangle\left\langle e_{q}, T\left(e_{j}\right)\right\rangle \\
& =\sum_{p \in I}\left\langle T\left(e_{i}\right), e_{p}\right\rangle\left\langle e_{p}, e_{j}\right\rangle+\sum_{q \in I}\left\langle e_{i}, e_{q}\right\rangle\left\langle e_{q}, T\left(e_{j}\right)\right\rangle .
\end{aligned}
$$


Therefore it follows from the last equation that

$$
2 \sum_{p \in I}\left\langle T\left(e_{i}\right), e_{p}\right\rangle\left\langle e_{p}, T\left(e_{j}\right)\right\rangle=\left\langle T\left(e_{i}\right), e_{j}\right\rangle+\left\langle e_{i}, T\left(e_{j}\right)\right\rangle \quad(i, j \in I) .
$$

Let $a_{i j}=\left\langle T\left(e_{j}\right), e_{i}\right\rangle$ for all $i, j \in I$, then the last equation implies that

$$
2 \sum_{p \in I} a_{p i} a_{p j}^{*}=a_{j i}+a_{i j}^{*} \quad(i, j \in I)
$$

This means that $2 A^{t} A^{*}=A^{t}+A^{*}$. Conversely, if $2 A^{t} A^{*}=A^{t}+A^{*}$, it is easy to see that $T$ satisfies $(\diamond)$.

Corollary 2.4. Let $\mathcal{H}$ be a Hilbert $C^{*}$-module over a unital $C^{*}$-algebra $\mathcal{A}$. An $\mathcal{A}$-linear operator $T: \mathcal{H} \rightarrow \mathcal{H}$ satisfies $(\diamond)$, if and only if $T$ is adjointable and satisfies the equation $2 T^{*} T=T+T^{*}$.

Proof. Suppose that $T$ satisfies $(\diamond)$. From $(5)$ we have

$$
2\left(A^{*}\right)^{t} A=A+\left(A^{*}\right)^{t} .
$$

Define the $\mathcal{A}$-linear operator $S: \mathcal{H} \rightarrow \mathcal{H}$ by the matrix $\left(A^{*}\right)^{t}$. Then trivially $\left\langle S\left(e_{j}\right), e_{i}\right\rangle=a_{j i}^{*}$ for all $i, j \in I$ and it follows that $\left\langle e_{j}, S\left(e_{i}\right)\right\rangle=a_{i j}=\left\langle T\left(e_{j}\right), e_{i}\right\rangle$ for all $i, j \in I$. From $\mathcal{A}$-linearity of $T$ and $S$, we get

$$
\langle T(x), y\rangle=\langle x, S(y)\rangle \quad(x, y \in \mathcal{H})
$$

So $T$ is adjointable with $T^{*}=S$. From (6) we get $2 T^{*} T=T+T^{*}$. Conversely, if $T$ is an adjointable $\mathcal{A}$-linear operator such that $2 T^{*} T=T+T^{*}$, then it satisfies $(\diamond)$.

Remark 2.5. Suppose that the mappings $T$ and $S$ satisfy $(\diamond)$ and $\langle T(x), S(y)\rangle=0$ for all $x, y \in \mathcal{H}$, then for any complex number $\lambda=(r, \theta)$ with $r=2 \cos \theta$, the adjointable $\mathcal{A}$-linear mapping $\lambda T+S$ satisfies $(\diamond)$. Also if the mapping $T$ satisfies $(\diamond)$, then $I-T$ satisfies $(\diamond)$.

Example 2.6. The Hilbert space $\ell^{2}$ is a Hilbert $\mathbb{C}$-module. The $\mathbb{C}$-linear operator $T: \ell^{2} \rightarrow \ell^{2}$ defined by

$$
T\left(x_{1}, x_{2}, x_{3}, \ldots\right)=\left(0, x_{2}, x_{3}, \ldots\right)
$$

for all $x=\left(x_{1}, x_{2}, x_{3}, \ldots\right) \in \ell^{2}$, satisfies $(\diamond)$, also $\|T\|<1$ and $T=T^{*}$. 
Example 2.7. Let $T: \mathbb{C}^{2} \rightarrow \mathbb{C}^{2}$ be a $\mathbb{C}$-linear operator, corresponding with the complex matrix $A=\left[a_{i j}\right]$. $T$ satisfies $(\diamond)$, if and only if the complex numbers $a_{i j}$ satisfy the following equations:

$$
\begin{gathered}
\left|a_{11}-\frac{1}{2}\right|^{2}+\left|a_{21}\right|^{2}=\frac{1}{4}, \quad\left|a_{22}-\frac{1}{2}\right|^{2}+\left|a_{12}\right|^{2}=\frac{1}{4} \\
\left|a_{12}\right|=\left|a_{21}\right| \leq \frac{1}{2}, \quad\left|a_{11}-\frac{1}{2}\right|=\left|a_{22}-\frac{1}{2}\right| \leq \frac{1}{2}
\end{gathered}
$$

Remark 2.8. Let $\alpha, \beta, \gamma \in \mathbb{C}$ be nonzero complex numbers and $T: \mathcal{H} \rightarrow \mathcal{H}$ be a mapping satisfing

$$
\alpha\langle T(x), T(y)\rangle=\beta\langle T(x), y\rangle+\gamma\langle x, T(y)\rangle \quad(x, y \in \mathcal{H})
$$

It is easy to see that $T$ is an adjointable $\mathcal{A}$-linear mapping with $\|T\| \leq \frac{|\beta|+|\gamma|}{|\alpha|}$. Moreover it follows that $\alpha T^{*} T=\beta T+\gamma T^{*}$ or equivalently $\left(\frac{\alpha}{\beta} T^{*}-I\right)\left(\frac{\alpha}{\gamma} T-I\right)=$ $I$. Thus if $\frac{\alpha}{\gamma} T-I$ is surjective, then it is invertible in $L(\mathcal{H})$ with $\left(\frac{\alpha}{\gamma} T-I\right)^{-1}=$ $\frac{\alpha}{\beta} T^{*}-I$.

In particular, if the mapping $T$ satisfies $\left(\diamond^{\prime}\right)$ with $\alpha=\beta=\gamma=1$, then $I-T$ is inner product preserving and so is an isometry.

\section{Stability}

In this section, we prove the generalized Hyers-Ulam stability of the equation $(\diamond)$.

Theorem 3.1. Let $\mathcal{H}$ be a Hilbert $C^{*}$-module over a unital $C^{*}$-algebra $\mathcal{A}$ and $\varphi: \mathcal{H} \times \mathcal{H} \rightarrow[0, \infty)$ be an control function such that

$$
\lim _{n \rightarrow \infty} 2^{2 n} \varphi\left(\frac{x}{2^{n}}, \frac{y}{2^{n}}\right)=0
$$

for all $x, y \in \mathcal{H}$. Assume that there is $0<L<1$ such that

$$
\psi\left(\frac{x}{2}\right) \leq \frac{L}{2} \psi(x)
$$

for all $x \in \mathcal{H}$, where

$$
\psi(x)=(4 \varphi(x, x)+2 \varphi(2 x, x)+2 \varphi(x, 2 x)+\varphi(2 x, 2 x))^{\frac{1}{2}} .
$$


If a function $f: \mathcal{H} \rightarrow \mathcal{H}$ satisfies the inequality

$$
\|2\langle f(x), f(y)\rangle-\langle f(x), y\rangle-\langle x, f(y)\rangle\| \leq \varphi(x, y)
$$

for all $x, y \in \mathcal{H}$, then there exists a unique adjointable mapping $T: \mathcal{H} \rightarrow \mathcal{H}$ such that

$$
\|f(x)-T(x)\| \leq \frac{\sqrt{2} L}{4(1-L)} \psi(x)
$$

for all $x \in \mathcal{H}$.

Proof. Replacing $x$ by $2 x$ in (10), we get

$$
\|2\langle f(2 x), f(y)\rangle-\langle f(2 x), y\rangle-2\langle x, f(y)\rangle\| \leq \varphi(2 x, y) .
$$

From (10) and (12), we have

$$
\|2\langle f(2 x)-2 f(x), f(y)\rangle-\langle f(2 x)-2 f(x), y\rangle\| \leq 2 \varphi(x, y)+\varphi(2 x, y) .
$$

Replacing $y$ by $2 y$ in (13), we get

$$
\|2\langle f(2 x)-2 f(x), f(2 y)\rangle-2\langle f(2 x)-2 f(x), y\rangle\| \leq 2 \varphi(x, 2 y)+\varphi(2 x, 2 y) .
$$

From (13) and (14), we have

$$
\|2\langle f(2 x)-2 f(x), f(2 y)-2 f(y)\rangle\| \leq \psi(x)^{2} .
$$

Letting $x=y$ in (15), we obtain

$$
\|f(2 x)-2 f(x)\|^{2}=\left\||f(2 x)-2 f(x)|^{2}\right\| \leq \frac{1}{2} \psi(x)^{2} .
$$

and so

$$
\|f(2 x)-2 f(x)\| \leq \frac{\sqrt{2}}{2} \psi(x)
$$

Let $X=\{g: \mathcal{H} \rightarrow \mathcal{H}, g(0)=0\}$ and define $d: X \times X \rightarrow[0, \infty]$ by

$$
d(g, h)=\inf \{\alpha \geq 0:\|g(x)-h(x)\| \leq \alpha \psi(x), \forall x \in \mathcal{H}\} \quad(g, h \in X) .
$$

Define $J: X \rightarrow X$ by $J(g)(x)=2 g\left(\frac{x}{2}\right)$ for each $x \in \mathcal{H}$. Then $(X, d)$ is a complete generalized metric space and from (8) it follows that $J$ is a strictly contractive mapping on $X$ with the Lipschitz constant $L=\frac{1}{2}$. From (8) and (16) we have

$$
\|J(f)(x)-f(x)\|=\left\|2 f\left(\frac{x}{2}\right)-f(x)\right\|=2\left\|f\left(\frac{x}{2}\right)-\frac{f(x)}{2}\right\| \leq \frac{\sqrt{2}}{4} L \psi(x)
$$


for each $x \in \mathcal{H}$. This means that $d(J(f), f) \leq \frac{\sqrt{2}}{4} L$. Therefore, by Proposition $1.1, J$ has a unique fixed point in the set $X_{0}=\{g \in X: d(f, g)<\infty\}$. Let $T: \mathcal{H} \rightarrow \mathcal{H}$ be the unique fixed point of $J$. We have $\lim _{n}\left(J^{n}(f), T\right)=0$, so $T$ is defined by

$$
T(x):=\lim _{n \rightarrow \infty} 2^{n} f\left(\frac{x}{2^{n}}\right) \quad(x \in \mathcal{H}) .
$$

On the other hand, we have $d(f, J(f)) \leq \frac{\sqrt{2}}{4} L$ and $J(T)=T$, then

$$
d(f, T) \leq d(f, J(f))+d(J(f), J(T)) \leq \frac{\sqrt{2}}{4} L+L d(f, T) .
$$

So

$$
d(f, T) \leq \frac{\sqrt{2} L}{4(1-L)},
$$

which implies the inequality (11).

It is easy to see that for all $x, y \in \mathcal{H}$, we have

$$
\begin{aligned}
& \lim _{n \rightarrow \infty}\left\langle 2^{n} f\left(\frac{x}{2^{n}}\right), y\right\rangle=\langle T(x), y\rangle, \\
& \lim _{n \rightarrow \infty}\left\langle x, 2^{n} f\left(\frac{y}{2^{n}}\right)\right\rangle=\langle x, T(y)\rangle .
\end{aligned}
$$

Since for every $x \in \mathcal{H}$ the sequence $\left\{2^{n} f\left(\frac{x}{2^{n}}\right)\right\}$ is convergent, so it is bounded. Therefore for every $x \in \mathcal{H}$ there exists $K_{x}>0$ such that $\left\|2^{n} f\left(\frac{x}{2^{n}}\right)\right\| \leq K_{x}$ for all $n \in \mathbb{N}$. Thus

$$
\begin{aligned}
& \lim _{n \rightarrow \infty}\left\|\left\langle 2^{n} f\left(\frac{x}{2^{n}}\right), 2^{n} f\left(\frac{y}{2^{n}}\right)\right\rangle-\langle T(x), T(y)\rangle\right\| \\
& =\lim _{n \rightarrow \infty} \|\left\langle 2^{n} f\left(\frac{x}{2^{n}}\right), 2^{n} f\left(\frac{y}{2^{n}}\right)\right\rangle-\left\langle 2^{n} f\left(\frac{x}{2^{n}}\right), T(y)\right\rangle \\
& +\left\langle 2^{n} f\left(\frac{x}{2^{n}}\right), T(y)\right\rangle-\langle T(x), T(y)\rangle \| \\
& =\lim _{n \rightarrow \infty}\left\|\left\langle 2^{n} f\left(\frac{x}{2^{n}}\right), 2^{n} f\left(\frac{y}{2^{n}}\right)-T(y)\right\rangle+\left\langle 2^{n} f\left(\frac{x}{2^{n}}\right)-T(x), T(y)\right\rangle\right\| \\
& \leq \lim _{n \rightarrow \infty}\left(\left\|2^{n} f\left(\frac{x}{2^{n}}\right)\right\|\left\|2^{n} f\left(\frac{y}{2^{n}}\right)-T(y)\right\|+\left\|2^{n} f\left(\frac{x}{2^{n}}\right)-T(x)\right\|\|T(y)\|\right) \\
& \leq \lim _{n \rightarrow \infty}\left(K_{x}\left\|2^{n} f\left(\frac{y}{2^{n}}\right)-T(y)\right\|+\left\|2^{n} f\left(\frac{x}{2^{n}}\right)-T(x)\right\|\|T(y)\|\right)=0
\end{aligned}
$$

for all $x, y \in \mathcal{H}$. This shows that

$$
\lim _{n \rightarrow \infty}\left\langle 2^{n} f\left(\frac{x}{2^{n}}\right), 2^{n} f\left(\frac{y}{2^{n}}\right)\right\rangle=\langle T(x), T(y)\rangle \quad(x, y \in \mathcal{H}) .
$$


It follows from (7), (18), (19) and (20) that

$$
\begin{aligned}
& \|2\langle T(x), T(y)\rangle-\langle T(x), y\rangle-\langle x, T(y)\rangle\| \\
& =\lim _{n \rightarrow \infty} 2^{2 n}\left\|2\left\langle f\left(\frac{x}{2^{n}}\right), f\left(\frac{y}{2^{n}}\right)\right\rangle-\left\langle f\left(\frac{x}{2^{n}}\right), \frac{y}{2^{n}}\right\rangle-\left\langle\frac{x}{2^{n}}, f\left(\frac{y}{2^{n}}\right)\right\rangle\right\| \\
& \leq \lim _{n \rightarrow \infty} 2^{2 n} \varphi\left(\frac{x}{2^{n}}, \frac{y}{2^{n}}\right)=0
\end{aligned}
$$

for all $x, y \in \mathcal{H}$. Whence

$$
2\langle T(x), T(y)\rangle=\langle T(x), y\rangle+\langle x, T(y)\rangle
$$

for all $x, y \in \mathcal{H}$ and so by Corollary (2.4), $\mathrm{T}$ is a adjointable $\mathcal{A}$-linear mapping.

To see the uniqueness of $T$, let $T^{\prime}: \mathcal{H} \rightarrow \mathcal{H}$ be another adjointable $\mathcal{A}$-linear mapping satisfying (11). Then

$$
\begin{aligned}
\left\|T(x)-T^{\prime}(x)\right\| & =2^{n}\left\|T\left(\frac{x}{2^{n}}\right)-T^{\prime}\left(\frac{x}{2^{n}}\right)\right\| \\
& \leq 2^{n}\left(\left\|T\left(\frac{x}{2^{n}}\right)-f\left(\frac{x}{2^{n}}\right)\right\|+\left\|f\left(\frac{x}{2^{n}}\right)-T^{\prime}\left(\frac{x}{2^{n}}\right)\right\|\right) \\
& \leq 2^{n} \frac{\sqrt{2} L}{2(1-L)} \psi\left(\frac{x}{2^{n}}\right) \\
& \leq \frac{\sqrt{2} L}{2(1-L)} L^{n} \psi(x)
\end{aligned}
$$

which tends to zero as $n \rightarrow \infty$ for all $x \in \mathcal{H}$. This completes the proof.

The following Theorem can be proved in a similar way as Theorem 3.1.

Theorem 3.2. Let $\mathcal{H}$ be a Hilbert $C^{*}$-module over a unital $C^{*}$-algebra $\mathcal{A}$ and $\varphi: \mathcal{H} \times \mathcal{H} \rightarrow[0, \infty)$ be an control function such that

$$
\lim _{n \rightarrow \infty} \frac{\varphi\left(2^{n} x, 2^{n} y\right)}{2^{2 n}}=0
$$

for all $x, y \in \mathcal{H}$. Assume that there is $0<L<1$ such that

$$
\psi(2 x) \leq 2 L \psi(x)
$$

for all $x \in \mathcal{H}$, where

$$
\psi(x)=(4 \varphi(x, x)+2 \varphi(2 x, x)+2 \varphi(x, 2 x)+\varphi(2 x, 2 x))^{\frac{1}{2}} .
$$


If a function $f: \mathcal{H} \rightarrow \mathcal{H}$ satisfies the inequality

$$
\|2\langle f(x), f(y)\rangle-\langle f(x), y\rangle-\langle x, f(y)\rangle\| \leq \varphi(x, y)
$$

for all $x, y \in \mathcal{H}$, then there exists a unique adjointable mapping $T: \mathcal{H} \rightarrow \mathcal{H}$ such that

$$
\|f(x)-T(x)\| \leq \frac{\sqrt{2}}{4(1-L)} \psi(x) \quad(x \in \mathcal{H}) .
$$

The next result follows from Theorem 3.1, where $\varphi(x, y)=\|x-y\|^{p}$ for all $x, y \in \mathcal{H}$.

Corollary 3.3. Let $\mathcal{H}$ be a Hilbert $C^{*}$-module over a unital $C^{*}$-algebra $\mathcal{A}$. If a function $f: \mathcal{H} \rightarrow \mathcal{H}$ satisfies the inequality

$$
\|2\langle f(x), f(y)\rangle-\langle f(x), y\rangle-\langle x, f(y)\rangle\| \leq\|x-y\|^{p}
$$

for all $x, y \in \mathcal{H}$ and some $p>2$, then there exists a unique adjointable mapping $T: \mathcal{H} \rightarrow \mathcal{H}$ such that

$$
\|f(x)-T(x)\| \leq \frac{\sqrt{2}}{\sqrt{2^{p}}-2}\|x\|^{\frac{p}{2}} \quad(x \in \mathcal{H}) .
$$

The next result follows from Theorem 3.2, where $\varphi(x, y)=\varepsilon$.

Corollary 3.4. Let $\mathcal{H}$ be a Hilbert $C^{*}$-module over a unital $C^{*}$-algebra $\mathcal{A}$. If a function $f: \mathcal{H} \rightarrow \mathcal{H}$ satisfies the inequality

$$
\|2\langle f(x), f(y)\rangle-\langle f(x), y\rangle-\langle x, f(y)\rangle\| \leq \varepsilon
$$

for all $x, y \in \mathcal{H}$, then there exists a unique adjointable mapping $T: \mathcal{H} \rightarrow \mathcal{H}$ such that

$$
\|f(x)-T(x)\| \leq \frac{3 \sqrt{2 \varepsilon}}{2} \quad(x \in \mathcal{H}) .
$$

Example 3.5. Consider the Hilbert $\mathbb{C}$-module $\ell^{2}$. Let $\phi: \ell^{2} \rightarrow \mathbb{C}$ be an arbitrary bounded function such that $|\phi(x)| \leq\|x\|$ for all $x \in \ell^{2}$, where $\|x\|=\left(\sum_{n=1}^{\infty}\left|x_{n}\right|^{2}\right)^{\frac{1}{2}}$. Define the mapping $f: \ell^{2} \rightarrow \ell^{2}$ by

$$
f\left(x_{1}, x_{2}, x_{3}, \ldots\right)=\left(\phi(x) x_{1}, x_{2}, x_{3}, \ldots\right)
$$


for all $x=\left(x_{1}, x_{2}, x_{3}, \ldots\right) \in \ell^{2}$. Then

$$
\begin{aligned}
& |2\langle f(x), f(y)\rangle-\langle f(x), y\rangle-\langle x, f(y)\rangle| \\
& =\mid 2 \phi(x) x_{1} \overline{\phi(y) y_{1}}+2 \sum_{n=2}^{\infty} x_{n} \overline{y_{n}} \\
& -\phi(x) x_{1} \overline{y_{1}}-\sum_{n=2}^{\infty} x_{n} \overline{y_{n}}-x_{1} \overline{\phi(y) y_{1}}-\sum_{n=2}^{\infty} x_{n} \overline{y_{n}} \mid \\
& =\left|\left(x_{1} \overline{y_{1}}\right)(2 \phi(x) \overline{\phi(y)}-\phi(x)-\overline{\phi(y)})\right| \\
& \leq\left|x_{1} \| y_{1}\right|(2|\phi(x)||\phi(y)|+|\phi(x)|+|\phi(y)|) \\
& \leq\|x\|\|y\|(2\|x\|\|y\|+\|x\|+\|y\|) \\
& =2\|x\|^{2}\|y\|^{2}+\|x\|^{2}\|y\|+\|x\|\|y\|^{2}
\end{aligned}
$$

for all $x, y \in \ell^{2}$. Thus if the control function is defined by

$$
\varphi(x, y)=2\|x\|^{2}\|y\|^{2}+\|x\|^{2}\|y\|+\|x\|\|y\|^{2} \quad\left(x, y \in \ell^{2}\right),
$$

then we have $\lim _{n \rightarrow \infty} 2^{2 n} \varphi\left(2^{-n} x, 2^{-n} y\right)=0$ and $\psi(x)=\left(72\|x\|^{4}+48\|x\|^{3}\right)^{\frac{1}{2}}$. Also for constant $0<L=\frac{\sqrt{2}}{2}<1$, we have $\psi\left(\frac{x}{2}\right) \leq \frac{L}{2} \psi(x)$ for all $x \in \ell^{2}$. Then the function $T: \ell^{2} \rightarrow \ell^{2}$ defined by

$$
T\left(x_{1}, x_{2}, x_{3}, \ldots\right)=\left(0, x_{2}, x_{3}, \ldots\right)
$$

for all $x=\left(x_{1}, x_{2}, x_{3}, \ldots\right) \in \ell^{2}$, is the unique adjointable mapping fulfilling the condition (11) in Theorem 3.1.

\section{References}

[1] D. Bakić, B. Guljaš, Hilbert $C^{*}$-modules over $C^{*}$-algebras of Compact Operators, Acta Sci. Math. (Szeged), 68 (2002), 249-269.

[2] J.B. Diaz, B. Margolis, A fixed point theorem of the alternative for the contractions on generalized complete metric space, Bull. Amer. Math. Soc., 74 (1968), 305-309.

[3] I. Kaplansky, Modules Over Operator Algebras, Amer. J. Math., 75 (1953), 839-858.

[4] E.C. Lance, Hilbert $C^{*}$-modules: A Toolkit for Operator Algebraists, Cambridge University Press, Cambridge, (1995).

[5] G. Landi, A. Pavlov, On Orthogonal Systems in Hilbert $C^{*}$-modules, Journal of Operator Theory, 68, No. 2 (2012), 487-500.

[6] V. Radu, The fixed point alternative and stability of functional equations, Fixed Point Theory, 4 (2003), 91-96. 